\title{
Salience of emotion in recall
}

\author{
K. T. STRONGMAN and P. N. RUSSELL \\ University of Canterbury, Christchurch, New Zealand
}

\begin{abstract}
A total of 183 subjects were run in a counterbalanced study designed to explore the relationship between emotion and recall. Results clearly establish that emotional material has a more prominent role in recall than has other material. Discussion is presented mainly in terms of Posner and Snyder's (1975) views and Bower's (1981) analysis of emotion and memory. It is concluded that the results support Posner's (1978) suggestion that emotion raises the level of alertness of the system and thereby enhances recall.
\end{abstract}

Strongman (1982) reported an investigation of emotion memory based on an adaptation of a procedure followed by Craik and Tulving (1975, Experiment 7). It involved the recall of emotional or neutral target words from sentence frames which themselves varied in emotionality. The results of that study indicated that the emotionality of stimulus material affects the ease of recall, although the nature of the relationship depends on the retrieval procedure used (free recall vs. cued recall). Some support was offered for Posner and Snyder's (1975) notion that emotion memory is dominant over item memory and is also a particular instance of general semantic memory.

Although Strongman (1982) reported some reasonably powerful effects, the study had shortcomings and also posed further questions concerning emotion and memory. One problem was that, of necessity, the cued recall condition always followed the measure of free recall, thus introducing a confounding of task order and task. One aim of the present study was to replicate Strongman (1982) in order to ensure that the results of the latter were not due to confounding.

More importantly, although the previous study showed that the emotionality of both target and sentence frame increased recall compared to neutral stimulus material, this in itself does not demonstrate that the category of emotional items is more important than any other category of items to be remembered. For example, memory of this type of verbal material may simply be a matter of the manner in which a target is integrated into its context. If this were so, the emotionality per se of the material would be of secondary concern. Were this argument to have force, it would suggest that emotion memory is merely an example of a more general phenomenon, namely, the relation of processes of the abstraction of generalized categorization and description provided in a recall situation. The prediction would then be that results similar to those of Strongman (1982) would be obtained with any other category of stimulus material.

Requests for reprints should be addressed to K. T. Strongman, Department of Psychology, University of Canterbury, Christchurch 1, New Zealand.
The main aim of the present study was to attempt a replication of the previous results with a simplified but more balanced and less confounded experimental design, while employing a control category to act as a further test of the salience (or other effects) of emotion in recall. The choice of another category of stimulus material was to some extent arbitrary. In the event, the category "animal" was selected, since there appeared to be similar degrees of difficulty in constructing targets and sentence frames which were or were not "animal" and ones which were or were not "emotional."

The experimental predictions were: (1) that the emotionality of the targets and sentence frames would affect free recall; (2a) that if, as Posner and Snyder (1975) suggest, there is a special category of emotion memory, then animal targets and sentence frames should, by comparison with emotional material, have little or no effect on free recall; or, alternatively, (2b) that if emotion memory is an example of a more general phenomenon, then recall of the "animal" material should be very similar to that of the "emotional" material.

\section{METHOD}

Subjects

A total of 183 students undertaking a Stage 1 psychology course served as subjects.

\section{Stimulus Materials}

Following the format used in Strongman (1982), 160 sentences were composed with a model length of 16 words each. Each took the form of a frame, and one word, always a noun, was designated as a target. Eighty of the sentences were designed to have emotional content, in the frame and/or in the target word, and 80 had an animal content, in the frame and/or in the target word.

Emotional sentences. The emotion sentences were presented in five blocks of four. Within each block there were two sentences with emotional frames and two sentences with neutral frames. Two emotional and two neutral target words were chosen so that each of the four words could be inserted into each of the four sentence frames without loss of meaning. For example, one set of 16 of the 80 emotion sentences was as follows (target words are italicized):

Emotional Frame 1. The young child's breast/nipple/face/hand was brutally cut by her mentally deranged father.

Emotional Frame 2. The high-speed drill slipped from the dentist's grasp and sank directly into the woman's breast/nipple/face/hand. 
Neutral Frame 1. The child looked at her breast/nipple/face/hand for a moment before switching off the light and going to sleep.

Neutral Frame 2. The woman with the swollen breast/nipple/face/ hand crossed the room and picked up the white handkerchief.

Thus, 10 sentences had emotional frames and 10 sentences had neutral frames. Each of these could be meaningfully completed with either of two emotional target words or either of two neutral target words.

In addition to this, for each balanced set of 16 sentences, an alternative to the target word was chosen such that, if it were inserted into the sentence, the result would be nonsensical. In the example given above, the word was concrete. All sentences were devised by one of the experimenters, and their relative emotionality and semantics discussed with the other experimenter and independent assessors.

Animal sentences. The 80 sentences with animal-related content were constructed in exactly the same manner as were the emotional sentences, involving full counterbalancing within each set of 16 . An example of one of the sets of 16 follows (target words italicized).

Animal Frame 1. As the breeder became busy with brush and comb, the dog/cat/owner/child seemed to puff up in pride.

Animal Frame 2. The vet made soothing noises to the dog/cat/owner/ child as he gave the lengthy injection.

Neutral Frame 1. The four young friends sat in the dining room and shared their meal with the dog/cat/owner/child.

Neutral Frame 2. As the man crossed the foyer of the expensive hotel, he glanced at the dog/cat/owner/child behind the desk.

In the case of these sentences, the alternative target word was "branch."

\section{Procedure}

Each sentence was put onto a slide. The sentence frame appeared with its target word missing, and its nonsensical alternative appeared beneath the sentence frame. The sentences were divided into eight blocks of 20 , each block being presented to a different group of subjects (group sizes ranged between 15 and 30 ).

Each sequence of slides was collated in a semibalanced way, according to each of the sets of 16 interrelated sentences, such that no group of subjects saw more than 1 sentence from each group of 16 , and such that each group of subjects saw only emotional or only animal sentences. The order of stimulus materials presented to each group is shown in Table 1 .

All subjects were instructed to complete each sentence by writing down whichever of the two target words they considered to be the more appropriate. After having completed 20 sentences, subjects were asked to list as many of the target words as they could remember from those they had chosen.

\section{Results and Analysis}

For the purposes of analyzing the results, the experimental design was redesignated as in Table 2. This involved considering the target word in terms of whether

Table 1

Order of Stimulus Slides Presented to Each Group

\begin{tabular}{lcccc}
\hline Target Word & \multicolumn{4}{c}{ Sentence Frame } \\
\hline & Emotion 1 & Emotion 2 & Neutral 1 & Neutral 2 \\
Emotion 1 & 1 & 2 & 3 & 4 \\
Emotion 2 & 2 & 1 & 4 & 3 \\
Neutral 1 & 3 & 4 & 1 & 2 \\
Neutral 2 & 4 & 3 & 2 & 1 \\
& Animal 1 & Animal 2 & Neutral 1 & Neutral 2 \\
Animal 1 & 5 & 6 & 7 & 8 \\
Animal 2 & 6 & 5 & 8 & 7 \\
Neutral 1 & 7 & 8 & 5 & 6 \\
Neutral 2 & 8 & 7 & 6 & 5 \\
\hline
\end{tabular}

Note-Each cell contains the number of the group which was presented with the relevant sentences. There were 5 sentences within each cell, so that each subject saw 20 sentences.
Table 2

Redesignation of Experimental Design

\begin{tabular}{lcc}
\hline & \multicolumn{2}{c}{ Target } \\
\cline { 2 - 3 } Frame & Positive & Neutral \\
\hline Same as target & Emotional & \\
Different from target & Groups 1 \& 2 & Groups 1 \& 2 \\
& Groups 3 \& 4 & Groups 3 \& 4 \\
Same as target & Animal & \\
Different from target & Groups 5 \& 6 & Groups 5 \& 6 \\
\hline
\end{tabular}

Table 3

Overall Means, with the Numbers in Each Group in Parentheses

\begin{tabular}{ccc}
\hline & \multicolumn{2}{c}{ Target } \\
\cline { 2 - 3 } Frame & Positive & Neutral \\
\hline & Emotional \\
Positive & $5.78(50)$ & $6.62(32)$ \\
Neutral & $3.91(32)$ & $4.04(50)$ \\
& Animal & \\
Positive & $4.52(56)$ & $4.24(45)$ \\
Neutral & $4.33(45)$ & $3.98(56)$ \\
\hline
\end{tabular}

it was congruent in kind to its sentence frame (e.g. emotional target, emotional frame) or different in kind from its sentence frame (e.g., emotional target, neutral frame). Also, since the experimental conditions were essentially duplicated for Groups 1 and 2, 3 and 4, 5 and 6, and 7 and 8 , the two sets of data which derived from these groups were collapsed into composite means. Following this procedure, overall means are shown in Table 3.

Following the redesignated design presented in Table 2, the data were tested by a three-way ANOVA, with repeated measures on one factor (target type), and using an unweighted means analysis (Kirk, 1968).

The emotion/animal main effect was significant $[F(1,179)=17.38, p<.01]$, confirming the effect displayed in Table 3 for more words to be recalled from emotion than from animal conditions. Neither the target nor target frame relation main effects were significant. However, all interactions were significant. In particular, the emotion/animal $\times$ target $[F(1,179)=5.69, p<.05]$ and emotion/animal $\times$ target $\times$ target/frame relation $[F(1,179)=35.74, p<.01]$ effects were significant. Clearly, the effects of target and target/frame relations were different for the emotion and animal conditions.

These results necessitated separate two-way unweighted means ANOVAS on the emotion and animal data, with repeated measures on the target factor.

Animal data. Neither the type of animal target nor the type of animal frame had any significant effect on the recall data deriving from the animal sentences.

Emotion data. Two-way analysis of the data from the emotional sentences revealed a strong and significant interaction between target type and target/frame relation $[F(1,80)=86.81, p<.001]$. For emotional targets recall was greater when the type of sentence frame was the same as that of the target (i.e., emotional) than when it was different (i.e., neutral). 
This interaction prompts consideration of two questions: (1) Is the greater recall in emotional frames as opposed to neutral frames true of both emotional targets and neutral targets? (2) Is there any difference between emotional and neutral targets within emotional frames and within neutral frames?

(1) Frame differences within target types. There was a significant difference in mean recall between emotional targets in emotional frames (5.78) and emotional targets in neutral frames $(3.91)[F(1,160)=24.534, p<.01]$. There was also a significant difference in mean recall between neutral targets in emotional frames (6.62) and neutral targets in neutràl frames $(4.04)[F(1,160)=47.70$, $p<.01]$.

(2) Target differences within frame conditions. There were no significant differences in mean recall between target types in either emotional or neutral frames.

\section{DISCUSSION}

The results of the present study can be summarized as follows: (1) More words were recalled in the emotion than in the animal conditions. (2) Within the animal conditions, manipulation of frame and target type had no significant effect on the number of words recalled. (3) Within the emotion conditions, more emotional targets were recalled from emotional than from neutral frames, and more neutral targets were recalled from emotional than from neutral frames. However, there were no differences in recall between emotional and neutral target types within either emotional or neutral frames.

These results clearly establish the salience of emotional material over animal material in recall. This adds support to Posner and Snyder's (1975) idea that emotion memory has special characteristics which set it apart from memory for material from other categories. Certainly, emotion memory does not appear to be a specific instance of a more general phenomenon.

Although the present results endorse those of Strongman (1982), they differ in one respect: The previous effect of emotional sentence frames is clearly replicated, whereas the target effect is not. The reason for this is not readily apparent, but may depend on the degree of congruence between the emotional tone of the sentence frame and that of its target. However, post hoc inspection of this demonstrated complete congruence between emotional targets and emotional frames. It is also worth not- ing that all of the emotional material in the present study was negative in tone. This replicates exactly the conditions which were used in the strong emotional material in Strongman (1982).

In recall experiments it is frequently the case that some items are recalled at the expense of others. However, this has not occurred in the present study. Recall from emotional frames is clearly superior to recall in the animal condition, regardless of type of animal frame or target. Emotional sentence frames have acted, as would be predicted from Posner and Synder (1975), as a kind of mnemonic, raising overall level of performance.

For the moment, rather than continue the discussion of Posner and Snyder's (1975) suggestion of two independent memory structures, it might be helpful to consider Bower's (1981) analysis. In these terms, with the emotional frames there could be the additional mnemonic aid of an emotion node. If this is so, the general context, extraneous thoughts, emotional state, and any other elaborated retrieval cues present at the time of recall should become linked, at least weakly, to an emotional node. This would occur via a sentence which ought to have stronger links to words representing specific instances which evoke the emotion, some of which would be target words.

Although such ideas might help to account for the present effects of emotional frames, they would also predict better recall of emotional targets than of neutral targets, independently of the type of frame. Perhaps a more plausible suggestion is that emotion raises the level of alertness (Posner, 1978) of the system so that responses are made on the basis of less complete information. Be this as it may, the present results, in conjunction with those of Strongman (1982), demonstrate that a significant factor in memory is the emotionality of the material, and also begin to suggest ways in which emotional events might be recalled.

\section{REFERENCES}

Bower, G. H. (1981). Mood \& Memory. American Psychologist, 36, 129-148.

Craik, F. I. M., \& Tulving, E. (1975). Depth of processing and the retention of words in episodic memory. Journal of Experimental Psychology: General, 104, 268-294.

KIRK, R. E. (1968). Experimental design: Procedures for the behavioral sciences. Belmont, CA: Brooks/Cole.

Posner, M. I. (1978). Chronometric Explorations of Mind. Hillsdale, NJ: Erlbaum.

Posner, M. I., \& SNyder, C. R. R. (1975). Attention and cognitive control. In R. L. Solso (Ed.), Information processing and cognition: The Loyola Symposium. Hillsdale, NJ: Erlbaum.

Strongman, K. T. (1982). Emotional influences on memory. Current Psychological Research, 2, 69-74. 